Research Paper

\title{
The Effects of Antenatal Corticosteroids on Short- and Long-Term Outcomes in Small-for-Gestational-Age Infants
}

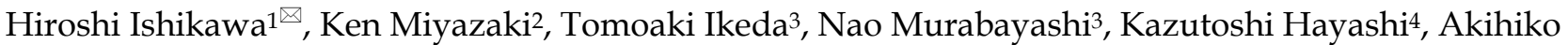
$\mathrm{Kai}^{5}$, Kaoru Ishikawa ${ }^{6}$, Yoshihiro Miyamoto7, Kunihiro Nishimura7, Yumi Kono ${ }^{8}$, Satoshi Kusuda9, Masanori Fujimura ${ }^{10}$, Neonatal Research Network of Japan

1. Department of Obstetrics and Gynecology, Kanagawa Children's Medical Center

2. Department of Obstetrics and Gynecology, Japanese Red Cross Nagoya Daiichi Hospital

3. Department of Obstetrics and Gynecology, Mie University Graduate School of Medicine

4. Department of Obstetrics and Gynecology, Kochi Health Sciences Center

5. Department of Pediatrics, Aizenbashi Hospital

6. Endowed Chair for Regeneration of Medicine in Kuwana District, Suzuka university of medical science

7. Department of Preventive Medicine and Epidemiologic Informatics, National Cerebral and Cardiovascular Center

8. Department of Pediatrics, Jichi Medical University

9. Department of Neonatology, Maternal and Perinatal Center, Tokyo Women's Medical University

10. Department of Neonatology, Osaka Medical Center and Research Institute for Maternal and Child Health, Director of the Neonatal Research Network of Japan.

$\triangle$ Corresponding author: Hiroshi Ishikawa, M.D., Ph.D. Department of Obstetrics and Gynecology, Kanagawa Children's Medical Center, Yokohama, Japan; Hiroshi Ishikawa 2-138-4 Mutsukawa, Minami-ku, Yokohama 232-8555, Japan. hishikawa@kcmc.jp. Telephone: +81 45-711-2351, Fax: +81 45-716-5366

() 2015 Ivyspring International Publisher. Reproduction is permitted for personal, noncommercial use, provided that the article is in whole, unmodified, and properly cited. See http://ivyspring.com/terms for terms and conditions.

Received: 2015.01.07; Accepted: 2015.02.25; Published: 2015.03.20

\begin{abstract}
Aim: To evaluate the effect of antenatal corticosteroids (ANS) on short- and long-term outcomes in small-for-gestational age (SGA) infants.

Methods: A retrospective database analysis was performed. A total of 1,931 single infants (birth weight $<1,500 \mathrm{~g}$ ) born at a gestational age between 22 weeks and 33 weeks 6 days who were determined to be SGA registered in the Neonatal Research Network Database in Japan between 2003 and 2007 were evaluated for short-term outcome and long-term outcome.

Results: ANS was administered to a total of 719 infants (37\%) in the short-term outcome evaluation group and 344 infants (36\%) in the long-term outcome evaluation group. There were no significant differences between the ANS group and the no-ANS group for primary short-term outcome (adjusted odds ratio (OR) 0.73; 95\% confidence interval (Cl) 0.45-1.20; P-value 0.22) or primary long-term outcome (adjusted OR 0.69; 95\% Cl 0.40-1.17; P-value 0.17).

Conclusions: Our results show that ANS does not affect short- or long-term outcome in SGA infants when the birth weight is less than $1500 \mathrm{~g}$. This study strongly suggests that administration of ANS resulted in few benefits for preterm FGR fetuses.
\end{abstract}

Key words: fetal growth restriction; glucocorticoids; infant; infant mortality; premature birth; small for gestational age

\section{Introduction}

Antenatal corticosteroid (ANS) administration in women who are at risk for preterm labor reduces the incidence of neonatal respiratory distress syndrome [1], intraventricular hemorrhage (IVH), necrotizing enterocolitis (NEC), and neonatal mortality [2]. Although administration of ANS is the most effective intervention for risks associated with preterm birth, the effect in some subgroups is limited. The effects of 
ANS in multiple pregnancies, in cases of chorioamnionitis (CAM) [3], and in cases of growth-restricted fetuses [4] are unclear. In addition, there is considerable controversy about the effectiveness of ANS in fetal growth restriction (FGR) [5].

The Neonatal Research Network Database is the largest database of level III perinatal centers in Japan. The database includes infants with birth weights at or less than 1,500 g, herein referred to as very-low-birth-weight (VLBW) infants, who were treated at participating neonatal centers. Considering the nation-wide population of VLBW infants born in Japan during the study period, more than $50 \%$ of VLBW infants in Japan were registered in the registry [6]. This database contains the factors of the maternal course (administered ANS or not), short-term outcomes, and long-term outcomes.

In this study, we conducted a retrospective analysis of the effectiveness of ANS on the short- and

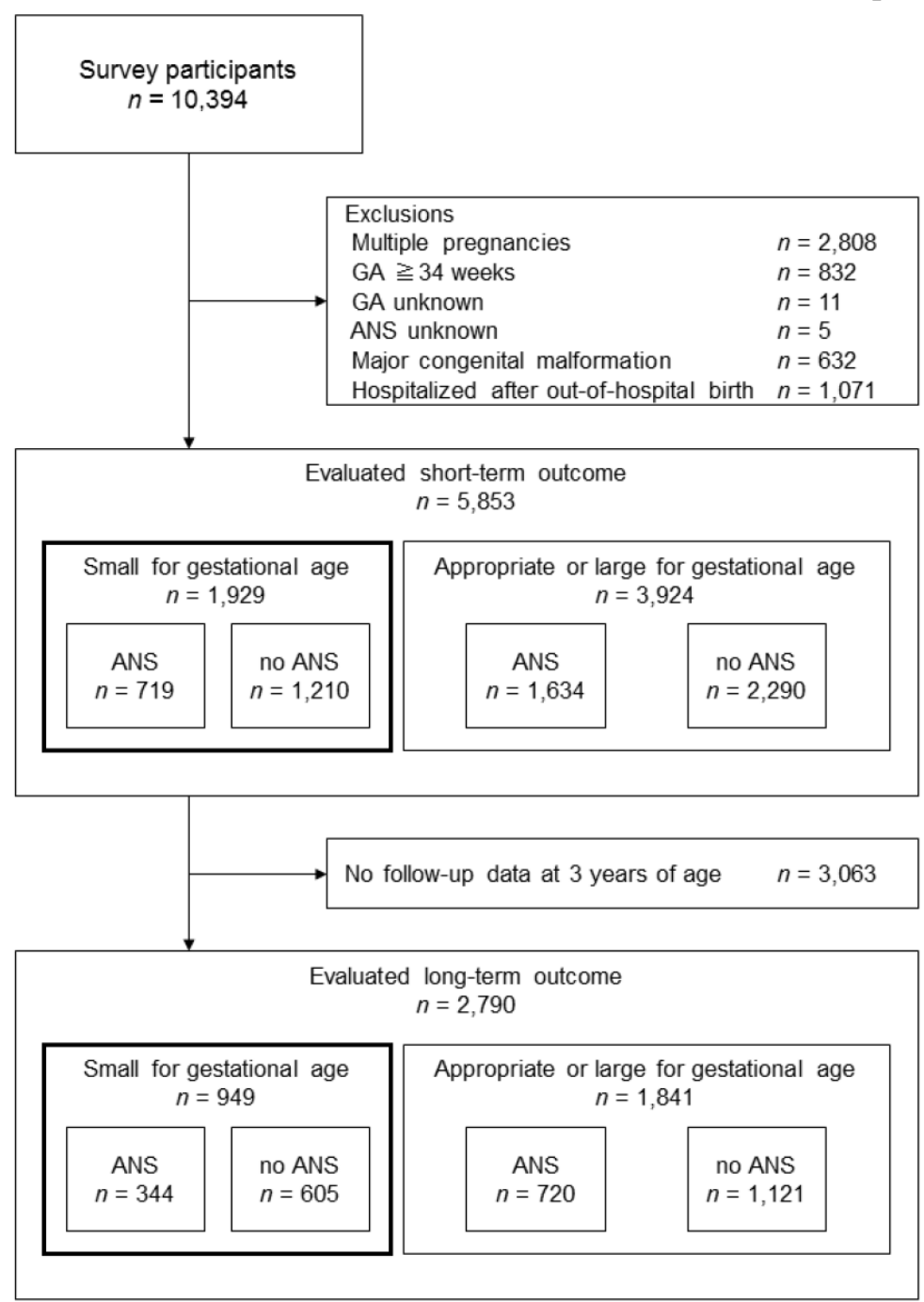

Figure 1. Study inclusion process for the short-term outcome evaluation group and the long-term outcome evaluation group. ANS, antenatal corticosteroids; GA, gestational age long-term outcomes in small-for-gestational-age (SGA) VLBW infants, which is a very high-risk group among preterm infants.

\section{Materials and Methods}

This was a retrospective analysis of the Neonatal Research Network Database created with a grant from the Ministry of Health, Labor, and Welfare of Japan in 2004. The 82 level III perinatal centers in Japan are registered in the database (listed in the Acknowledgments). Data include infants with birth weight less than $1,500 \mathrm{~g}$. Infants who were born alive but died in the delivery room were also included. The clinician's perspective on active treatment or withdrawal of care for preterm infants born at 22 and 23 weeks of gestation depended on the clinical status of the infants. After 23 weeks of gestation, most clinicians attempted to save the infants. All other factors were defined as reported previously [7].

There were 10,394 clinical cases between 2003 and 2007 (Fig. 1). Exclusion criteria were multiple pregnancies, 34 weeks of gestation or more, uncertain gestational age, uncertain administration of ANS, major congenital malformation, and hospitalization following an out-of-hospital birth. Short-term outcome evaluation was available in 5,853 cases. Of those, 3,063 cases dropped out of the follow-up before 3 years of age; therefore, long-term outcome was evaluated in 2,790 cases.

These cases were classified as either SGA or non-SGA. A birth weight below the 10th percentile for gestational age was classified as SGA. Birth weight for gestational age was determined using the percentile scale derived from the formula used in Japan [Itabashi, Fujimura, Kusuda, Tamura, Hayashi, et al. (2011) The new standard of birth weight for gestational age. J Jpn Pediatr Soc 114: 1271 -1293In Japanese].

ANS usage was defined as the administration of any corticosteroids to accelerate fetal lung maturity. ANS was provided based on the clinician's policy or perspective. The time from ANS administration to delivery and the type of corticosteroid used were not described in the database. It is inferred that betamethasone was used in most of the cases because betamethasone is the only drug that is officially recognized in the health insurance system of Japan for the acceleration of fetal lung maturation.

The primary short-term outcome was evaluated based on death occurring before 
discharge from a participating neonatal intensive care unit (death in NICU). Intraventricular hemorrhage $(\mathrm{IVH})$, periventricular leukomalacia (PVL), respiratory distress syndrome (RDS), chronic lung disease of prematurity (CLD), sepsis, late-onset adrenal insufficiency, symptomatic patent ductus arteriosus (PDA), and necrotizing enterocolitis (NEC) were also evaluated. IVH was defined as Papile grade I or more. The diagnosis of PVL was made based on either head ultrasound or cranial MRI scans performed at 2 weeks of age or later. RDS was diagnosed based on the clinical and radiographic findings. CLD was defined when an infant continued to receive supplemental oxygen on the 28th day after birth, and 36-week CLD was defined when an infant continued to receive supplemental oxygen at the 36th week based on postmenstrual age. PDA was diagnosed based on both the echocardiographic findings and clinical evidence of a volume overload due to a left-to-right shunt. NEC was defined according to a Bell classification [8] of stage II or greater.

For surviving VLBW infants, the follow-up protocol consisted of routine physical and neurological evaluations and developmental assessments at 3 years (36-42 months) of chronological age at each participating center, as reported previously [9]. The primary long-term outcome was evaluated based on death before 3 years of age or neurodevelopmental impairment (NDI). Infants with cerebral palsy $(\mathrm{CP})$, a development quotient $(\mathrm{DQ})<70$, and severe hearing impairment and visual impairment were designated as having neurodevelopmental impairment (NDI). CP was defined as a non-progressive central nervous system disorder characterized by abnormal muscle tone in at least one extremity and abnormal control of movement and posture [10]. DQ was determined using the Kyoto Scale of Psychological Development (KSPD) test applied by psychologists at each participating center [11]; when the DQ was < 70, the infant was judged as "delayed". Severe hearing impairment included the need for hearing aids. Visual impairment was defined as unilateral or bilateral blindness diagnosed by an ophthalmologist.

The results are expressed as the mean \pm SD or median (range). Statistical analysis was performed using the $\mathrm{Chi}^{2}$ test and t-test, as appropriate. Multivariable logistic regression analyses were performed to assess the effect of ANS on the short- and long-term outcomes. Odds ratios (OR) or confidence interval (CI) were adjusted for confounding variables, and 95\% confidence intervals were calculated. Multivariate logistic regression analysis was performed after adjusting for maternal age, parity, preeclampsia, preterm rupture of membranes (PROM), non-reassuring fetal status (NRFS), mode of delivery, gestational age of delivery, birth weight, gender of the infant, and histological CAM ( $\geq$ stage 2 according to Blanc's classification [12]).

Statistical analyses were performed using JMP, version 9.0.2J (SAS Institute, Cary, North Carolina, USA). Differences were considered to be statistically significant at $\mathrm{P}<0.05$.

All information about the infants was collected anonymously, and the stored data were unlinked from individual data. The protocol of this study was approved by the central internal review board at Tokyo Women's Medical University, where all data were collected and stored.

\section{Results}

\section{Short-term outcome}

A total of 10,394 infants were registered in the database between 2003 and 2007. In total, 4,541 infants were excluded. A total of 5,853 patients were evaluated for short-term outcomes. A total of 1,929 infants (33\%) were classified as SGA, and the 3,924 remaining infants were classified as appropriate or large for gestational age. ANS was administered to 719 of the SGA patients $(37 \%)$.

Table 1 compares the clinical characteristics of the ANS group and the no-ANS group in SGA infants. The incidence of preeclampsia was low, and the incidence of PROM was high in the ANS group. The ratio of cesarean section to vaginal birth was higher in the ANS group. Gestational age at delivery was earlier and birth weight was lower in the ANS group. Although RDS and CLD were more common in the ANS group, there was no difference in the primary short-term outcome (incidence of death before NICU discharge) and other short-term outcome factors between the ANS group and the no-ANS group based on univariate analysis.

To evaluate the effect of ANS on short-term outcome in SGA infants, further analysis was performed using logistic regression analysis. Table 2 shows the odds ratios of the ANS group compared with the no ANS group in SGA infants. Although the incidence of PVL demonstrated a trend toward a lower rate in the ANS group, the adjusted OR (95\% $\mathrm{CI})$ was $0.73(0.45-1.20)$ for the primary short-term outcome in the ANS group compared to the no-ANS group, significant effect of ANS was not observed in SGA infants.

\section{Long-term outcome}

A total of 3,063 infants were excluded because of the lack of follow-up data until 3 years of age. A total of 2,790 patients were evaluated for long-term outcome. Of those, 949 (34\%) infants were classified as SGA. ANS was administered to 344 of the SGA infants 
(36\%). The administration rate of ANS was similar to that of the SGA infants in the short-term evaluation group.

Table 3 shows a comparison of the clinical characteristics of the ANS group and the no-ANS group of SGA infants. The incidence of preeclampsia was low, and the incidence of PROM was high in the ANS group. Gestational age at delivery was lower, and birth weight was lower in the ANS group. Although severe hearing impairment was uncommon in the ANS group, there was no difference in the incidence of death before 3 years of age and other neurodevelopment impairment factors between the ANS group and the no-ANS group based on the univariate analysis.

To evaluate the effect of ANS on long-term outcome in SGA infants, further analysis was performed using logistic regression analysis. Table 4 shows the odds ratios of the ANS group compared to the no-ANS group of SGA infants. The adjusted OR $(95 \%$ CI) was $0.69(0.40-1.17)$ for the primary long-term outcomes in the ANS group compared to the no-ANS group; a significant long-term effect of ANS was not found in SGA infants.

Table 1. Comparison of the clinical characteristics and the results of the univariate analysis of the ANS group and the no-ANS group in the short-term outcome evaluation group of SGA infants $(n=1929)$

\begin{tabular}{|c|c|c|c|}
\hline & ANS $(n=719)$ & no ANS $(n=1210)$ & P value \\
\hline Maternal age & $32.0 \pm 4.9$ & $32.1 \pm 5.0$ & .50 \\
\hline Nulliparous & $437 / 719(61 \%)$ & $746 / 1210(62 \%)$ & .70 \\
\hline Preeclampsia & $318 / 719(44 \%)$ & $605 / 1209(50 \%)$ & $.0134^{*}$ \\
\hline Diabetes & $8 / 719(1 \%)$ & $25 / 1210(2 \%)$ & .11 \\
\hline PROM & $124 / 719(17 \%)$ & $118 / 1210(10 \%)$ & $<.0001^{*}$ \\
\hline NRFS & $309 / 719(43 \%)$ & $530 / 1209(44 \%)$ & .71 \\
\hline Cesarean section & $661 / 719(92 \%)$ & $1058 / 1210(87 \%)$ & $.0018^{*}$ \\
\hline GA at delivery & $29.1 \pm 2.6$ & $29.7 \pm 2.7$ & $<.0001^{*}$ \\
\hline Birth weight & $886 \pm 298$ & $959 \pm 313$ & $<.0001^{*}$ \\
\hline Male gender & $386 / 717(54 \%)$ & $615 / 1209(51 \%)$ & .21 \\
\hline Histological CAM ( $\geq$ stage 2) & $46 / 507(9 \%)$ & $46 / 838(5 \%)$ & $.0129^{*}$ \\
\hline Clinical CAM & $43 / 717(6 \%)$ & $75 / 1199(6 \%)$ & .82 \\
\hline $\begin{array}{l}\text { Death during NICU hospitaliza- } \\
\text { tion }\end{array}$ & $56 / 719(8 \%)$ & $92 / 1210(8 \%)$ & .88 \\
\hline IVH & $54 / 719(8 \%)$ & $99 / 1210(8 \%)$ & .60 \\
\hline PVL & $11 / 719(2 \%)$ & $28 / 1210(2 \%)$ & .23 \\
\hline RDS & $341 / 719(47 \%)$ & $510 / 1210(42 \%)$ & $.0241^{*}$ \\
\hline CLD & $194 / 719(27 \%)$ & $250 / 1210(21 \%)$ & $.0015^{*}$ \\
\hline Sepsis & $51 / 719(7 \%)$ & $75 / 1210(6 \%)$ & .44 \\
\hline Late-onset adrenal insufficiency & $53 / 719(7 \%)$ & $67 / 1210(5 \%)$ & .11 \\
\hline PDA & $175 / 719(24 \%)$ & $268 / 1210(22 \%)$ & .27 \\
\hline NEC & $13 / 719(2 \%)$ & $15 / 1210(1 \%)$ & .31 \\
\hline
\end{tabular}

ANS, antenatal corticosteroids; PROM, preterm rupture of membranes; NRFS, non-reassuring fetal status; GA, gestational age; CAM; chorioamnionitis; NICU, neonatal intensive care unit; $\mathrm{IVH}$, intraventricular hemorrhage; $\mathrm{PVL}$, periventricular leukomalacia; RDS, respiratory distress syndrome; CLD, chronic lung disease of prematurity; PDA, symptomatic patent ductus arteriosus; NEC, necrotizing enterocolitis.
Table 2. The results of multiple logistic analysis of the short-term outcome showing the adjusted odds ratio of the ANS group compared to the no-ANS group

\begin{tabular}{llll}
\hline & Adjusted OR† & 95\% CI & P value \\
\hline Death during NICU hospitalization & 0.73 & $0.45-1.20$ & .22 \\
IVH & 0.79 & $0.51-1.21$ & .28 \\
PVL & 0.44 & $0.17-1.03$ & .06 \\
RDS & 1.10 & $0.84-1.44$ & .48 \\
CLD & 1.18 & $0.87-1.62$ & .29 \\
Sepsis & 0.95 & $0.59-1.53$ & .84 \\
Late-onset adrenal insufficiency & 0.94 & $0.59-1.50$ & .80 \\
PDA & 1.01 & $0.76-1.34$ & .96 \\
NEC & 1.04 & $0.43-2.48$ & .92
\end{tabular}

ANS, antenatal corticosteroids; NICU, neonatal intensive care unit; IVH, intraventricular hemorrhage; PVL, periventricular leukomalacia; RDS, respiratory distress syndrome; CLD, chronic lung disease of prematurity; PDA, symptomatic patent ductus arteriosus; NEC, necrotizing enterocolitis.

†Adjusted for maternal age, parity, preeclampsia, PROM, NRFS, mode of delivery, gestational age at delivery, birth weight, gender of the infant, and histological CAM ( $\geq$ stage 2 )

Table 3. Comparison of the clinical characteristics and the results of the univariate analysis of the ANS group and the no-ANS group in the long-term outcome evaluation group of SGA infants $(n=949)$

\begin{tabular}{llll}
\hline & ANS $(\mathrm{n}=344)$ & no ANS $(\mathrm{n}=605)$ & P value \\
\hline Maternal age & $32.2 \pm 4.9$ & $32.1 \pm 5.0$ & .68 \\
Nulliparous & $215 / 344(63 \%)$ & $391 / 605(65 \%)$ & .51 \\
Preeclampsia & $135 / 344(39 \%)$ & $307 / 605(51 \%)$ & $.0006^{*}$ \\
Diabetes & $5 / 344(1 \%)$ & $15 / 605(2 \%)$ & .27 \\
PROM & $67 / 344(19 \%)$ & $55 / 605(9 \%)$ & $<.0001^{*}$ \\
NRFS & $148 / 344(43 \%)$ & $277 / 605(46 \%)$ & .41 \\
Cesarean section & $314 / 344(91 \%)$ & $534 / 605(88 \%)$ & .14 \\
GA at delivery & $28.7 \pm 2.7$ & $29.2 \pm 2.8$ & $.0025^{*}$ \\
Birth weight & $829 \pm 294$ & $900 \pm 320$ & $.0005^{*}$ \\
Male gender & $190 / 342(56 \%)$ & $316 / 604(52 \%)$ & .33 \\
Histological CAM (>stage 2) & $28 / 234(12 \%)$ & $23 / 436(5 \%)$ & $.0024^{*}$ \\
Clinical CAM & $24 / 344(7 \%)$ & $43 / 598(7 \%)$ & .90 \\
Death before 3 years of age & $59 / 344(17 \%)$ & $100 / 605(16 \%)$ & .81 \\
Neurodevelopment impair- & $66 / 285(23 \%)$ & $93 / 505(18 \%)$ & .11 \\
ment & & & .30 \\
Cerebral palsy & $19 / 278(7 \%)$ & $25 / 498(5 \%)$ & .12 \\
DQ<70 & $51 / 271(19 \%)$ & $70 / 486(14 \%)$ & $.0357^{*}$ \\
Severe hearing impairment & $0 / 277(0 \%)$ & $5 / 502(1 \%)$ & .64 \\
Visual impairment & $1 / 275(0 \%)$ & $3 / 490(1 \%)$ & .16 \\
Death before 3 years of age or & $125 / 344(36 \%)$ & $193 / 605(31 \%)$ & \\
NDI & & & \\
\hline ANS, antenatal corticosteroids; PROM, preterm rupture of membranes; NRFS, \\
non-reassuring fetal status; GA, gestational age; CAM; chorioamnionitis; DQ, \\
development quotient; NDI, neurodevelopmental impairment. & \\
& & & \\
& & &
\end{tabular}

Table 4. The results of multiple logistic analysis of the long-term outcome showing the adjusted odds ratio of the ANS group compared with the no ANS group

\begin{tabular}{|c|c|c|c|}
\hline & Adjusted OR† & $95 \% \mathrm{CI}$ & $P$ value \\
\hline Death before 3 years of age & 0.69 & $0.40-1.17$ & .17 \\
\hline Neurodevelopment impairment & 1.03 & $0.62-1.70$ & .90 \\
\hline Cerebral palsy & 1.12 & $0.41-2.96$ & .82 \\
\hline $\mathrm{DQ}<70$ & 1.08 & $0.63-1.85$ & .78 \\
\hline Severe hearing impairment & - & $0.00-1.42$ & .08 \\
\hline Visual impairment & 1.03 & $0.02-36.15$ & .99 \\
\hline Death before 3 years of age or NDI & 0.83 & $0.54-1.27$ & .39 \\
\hline
\end{tabular}




\section{Discussion}

Despite the established benefits of antenatal glucocorticoids for neonatal lung function and viability in normal-size premature infants, there is considerable controversy concerning the effectiveness of ANS in growth-restricted premature infants.

A large retrospective study found that the ANS affects fetal outcome even in cases of SGA. According to "The Vermont Oxford Network", ANS lowered the incidence of RDS, IVH, and perinatal death in both normal and growth-restricted newborns from 25 to 30 weeks gestation. The authors concluded that the benefits of ANS are not dependent on fetal growth [13].

In contrast, another study showed that ANS resulted in few benefits in cases of FGR. Elimian et al. found that there was no difference due to ANS in the incidence of RDS, IVH, PVL, NEC, and neonatal mortality in a retrospective study [4]. The case-control study of 62 pairs with growth-restriction due to placental insufficiency indicated that the survival rate without disability or handicap at 2 years corrected age was higher in the ANS group, but there was a statistically significant negative effect on physical growth in the long-term follow-up at school age. Schaap et al. concluded that the benefits from ANS for growth-restricted infants outweigh the possible adverse effects [14].

Several mechanisms of the ANS effect on FGR have been proposed. Early studies showed that that plasma cortisol levels in SGA fetuses were higher than in appropriate-for-gestational-age infants, suggesting that the ANS effect was attenuated [15]. Accordingly, the risk of RDS in FGR fetuses without ANS should decrease, but in fact, it increases [16]. A recent study of sheep fetuses with uterine artery ligation (an induced FGR model) showed no changes in surfactant protein gene expression when plasma cortisol concentrations were increased [17]. These studies suggest reactivity to corticosteroid concerning the pulmonary maturity deficits in FGR.

Administration of ANS for FGR might compromise cardiovascular function. A sheep study demonstrated that carotid blood flow decreased after the administration of betamethasone in an induced FGR model [18]. In contrast, human growth-restricted fetuses showed absent or reversed end-diastolic umbilical artery flow. Blood flow in umbilical arteries and veins increased after intramuscular injections of betamethasone in some cases [19]. Thus, the effects of ANS on the cardiovascular system and on prognosis warrant further investigation.

Our study demonstrated that ANS has no effect on the short- and long-term outcome in SGA infants. Mori et al. demonstrated that ANS improved short-term outcome using the same database [20], but their study did not delineate between SGA infants and non-SGA infants. This is the first large sample size retrospective study examining the effect of ANS on long-term outcomes in SGA infants.

The strength of this study is its large sample size. In addition, little distortion of the results due to inconsistent neonatal medical care is expected because the same health insurance system of Japan is provided to most inhabitants.

The limitations of our study are that it is a retrospective and multicenter study. The type of ANS and the days from ANS to delivery are not described in this database and are expected to vary. Although we excluded major malformation, the causes of SGA vary and are not described in the database, and it is expected that the response to ANS varies according to the cause of SGA. Another limitation is that the ANS administration rate was low. We suppose the reason for this was that ANS was not authorized in the public insurance institution of Japan until 2009; our study subjects were born between 2003 and 2007.

In summary, ANS is not effective to improve the short- or long-term outcome of SGA infants when the birth weight is less than $1500 \mathrm{~g}$. This study strongly suggests that the administration of ANS resulted in few benefits for preterm FGR fetuses. For randomization to be ethically justified, further study is needed to clarify ANS activity for FGR fetuses.

\section{Abbreviations}

ANS: antenatal corticosteroids; SGA: small-for-gestational age; IVH: intraventricular hemorrhage; NEC: necrotizing enterocolitis; CAM: chorioamnionitis; FGR: fetal growth restriction; VLBW: very-low-birth-weight; $\quad$ SGA: small-for-gestational-age; NDI: neurodevelopmental impairment; $\mathrm{CP}$ : cerebral palsy; DQ: development quotient

\section{Acknowledgements}

We wish to thank the institutions and representative physicians enrolled in the Neonatal Research Network Database in Japan, which include the following: Sapporo City General Hospital: S. Hattori; Kushiro Red Cross Hospital: A. Noro; Aomori Prefectural Central Hospital: T. Amizuka; Iwate Medical University: S. Chida; Sendai Red Cross Hospital: R. Takahashi; Akita Red Cross Hospital: H.Arai; Fukushima Medical University: T. Imamura; National Fukushima Hospital: N. Ujiie; University of Tsukuba: Y. Miyazono; Tsuchiura Kyodo General Hospital: J. Shimizu; Dokkyo Medical University: H. Suzumura; Jichi Medical University: Y. Kono; Saitama Children's Medical Center: M. Shimizu; Saitama Medical University Saitama Medical Center: T. Kunikata; Gunma 
Children's Medical Center: T. Fujiu; Kameda Medical Center: H. Sato; Tokyo Women's Medical University Yachiyo Medical Center: T. Kondo; Tokyo Metropolitan Bokuto Hospital: T. Watanabe; Showa University: M. Aizawa; Tokyo Women's Medical University: A. Uchiyama; Nihon University Itabashi Hospital: M. Makimoto; Teikyo University: J. Hoshi; Toho University: H. Yoda; Japan Red Cross Medical Center: Y. Kawakami; Aiiku Hospital: N. Ishii; National Center for Child Health and Development: Y. Ito; Kanagawa Children's Medical Center: H. Itani; Yokohama City University Medical Center: K. Seki; Tokai University: M. Nomura; Kitazato University: M. Nowatari; Yamanashi Prefectural Central Hospital: A. Nemoto; Nagaoka Red Cross Hospital: O. Nagata; Niigata City Hospital: Y. Nagayama; Nagano Children's Hospital: T. Nakamura; Shinshu University: M. Okada; Iida City Hospital: S. Nakata; National Nagano Hospital: E. Shimazaki; Saku General Hospital: T. Yoda; Toyama Prefectural Central Hospital: T. Hutatani; Ishikawa Prefectural Central Hospital: Y. Ueno; Fukui Prefectural Hospital: K. Iwai; Shizuoka Children's Hospital: Y. Nakazawa; Seirei Hamamatsu General Hospital: S. Oki; Nagoya Red Cross First Hospital: C. Suzuki; National Mie Hospital: M. Bonno; Gifu Prefectural Central Hospital: Y. Kawano; Otsu Red Cross Hospital: K. Nakamura; Kyoto Red Cross First Hospital: N. Mitsufuji; Osaka Medical Center and Research Institute for Maternal and Child Health: J. Shiraishi; Osaka City General Hospital: H. Ichiba; Takatsuki Hospital: H. Minami; Yodogawa Christian Hospital: H. Wada; Kansai Medical University: A. Ohashi; Aizenbashi Hospital: K. Sumi; Nara Medical University: Y. Takahashi; Wakayama Prefectural Medical University: T. Okutani; Hyogo Prefectural Kobe Children's Hospital: S. Yoshimoto; Tottori University: I. Nagata; Shimane Prefectural Central Hospital: E. Kato; Kurashiki Central Hospital: S. Watabe S; National Okayama Hospital: M. Kageyama; Hiroshima Prefectural Hospital: R. Fukuhara; Hiroshima City Hospital: M. Hayashitani; Yamaguchi Prefectural Medical Center: K. Hasegawa; National Kagawa Children's Hospital: A. Ohta; Kagawa University: T. Kuboi; Ehime Prefectural Central Hospital: S. Akiyoshi; Kochi Health Sciences Center: K. Kikkawa; Tokushima University: T. Saijo; St. Mary's Hospital: S. Shimokawa; Kitakyushu City Municipal Medical Center: N. Matsumoto; Kurume University: H. Kanda; Fukuoka University: E. Oota; National Kyushu Medical Center: G. Kanda; Kyushu University: M. Ochiai; National Nagasaki Medical Center: M. Aoki; Kumamoto City Hospital: Y. Kondo; Kumamoto University: M. Iwai; Oita Prefectural Hospital: K. Iida; Miyazaki University: T. Ikenoue; Kagoshima City Hospital: S. Ibara; Okinawa Chubu Hospital: M. Kohama.

\section{Competing Interests}

This study was partly supported by a grant from the Ministry of Health, Labor and Welfare, Japan.

\section{References}

1. Liggins GC, Howie RN. A controlled trial of antepartum glucocorticoid treatment for prevention of the respiratory distress syndrome in premature infants. Pediatr. 1972; 50: 515-525.

2. Crowley PA. Antenatal corticosteroid therapy: a meta-analysis of the randomized trials, 1972 to 1994. Am J Obstet Gynecol. 1995; 173: 322-335 [DOI:10.1016/0002-9378(95)90222-8].

3. Roberts D, Dalziel S. Antenatal corticosteroids for accelerating fetal lung maturation for women at risk of preterm birth. Cochrane Database Syst Rev. 2006; 19: CD004454; CD004454 [DOI:10.1002/14651858.CD004454.pub2].

4. Elimian A, Verma U, Canterino J, et al. Effectiveness of antenatal steroids in obstetric subgroups. Obstet Gynecol. 1999; 93: 174-179 [DOI:10.1016/S0029-7844(98)00400-1].

5. Morrison JL, Orgeig S. Antenatal glucocorticoid treatment of the growth-restricted fetus: benefit or cost? Reprod Sci. 2009; 16: 527-538 [DOI:10.1177/1933719109332821]

6. Kusuda S, Fujimura M, Uchiyama A, et al. Neonatal Research Network. Japan. trends in morbidity and mortality among very-low-birth-weight infants from 2003 to 2008 in Japan. Pediatr Res. 2012; 72: 531-538. [DOI: 10.1038/pr.2012.114.]

7. Kusuda S, Fujimura M, Sakuma I, et al.. Neonatal Research Network. Morbidity and mortality of infants with very low birth weight in Japan: center variation. Pediatr. 2006; 118: e1130-8 [DOI:10.1542/peds.2005-2724].

8. Bell MJ, Ternberg JL, Feigin RD, et al. Neonatal necrotizing enterocolitis. Therapeutic decisions based upon clinical staging. Ann Surg. 1978; 187: 1-7 [DOI:10.1097/00000658-197801000-00001].

9. Kono Y, Mishina J, Yonemoto N, et al. Outcomes of very-low-birthweight infants at 3 years of age born in 2003-2004 in Japan. Pediatr Int. 2011; 53: 1051-1058 [DOI:10.1111/j.1442-200X.2011.03480.x].

10. Bax MC. Terminology and classification of cerebral palsy. Dev Med Child Neurol. 1964; 6: 295-297 [DOI:10.1111/j.1469-8749.1964.tb10791.x].

11. Koyama $\mathrm{T}$, Osada $\mathrm{H}$, Tsujii $\mathrm{H}$, et al. Utility of the Kyoto scale of psychological development in cognitive assessment of children with pervasive developmental disorders. Psychiatry Clin Neurosci. 2009; 63: 241-243 [DOI:10.1111/j.1440-1819.2009.01931.x].

12. Blanc WA. Pathology of the placenta, membranes, and umbilical cord in bacterial, fungal, and viral infections in man. Monogr Pathol. 1981; 22: 67-132.

13. Bernstein IM, Horbar JD, Badger GJ, et al. Morbidity and mortality among very-low-birth-weight neonates with intrauterine growth restriction. The Vermont Oxford Network. Am J Obstet Gynecol. 2000; 182: 198-206 [DOI:10.1016/S0002-9378(00)70513-8].

14. Schaap $\mathrm{AH}$, Wolf $\mathrm{H}$, Bruinse $\mathrm{HW}$, et al. Effects of antenatal corticosteroid administration on mortality and long-term morbidity in early preterm, growth-restricted infants. Obstet Gynecol. 2001; 97: 954-960 [DOI:10.1016/S0029-7844(01)01343-6].

15. Economides DL, Nicolaides KH, Linton EA, et al. Plasma cortisol and adrenocorticotropin in appropriate and small for gestational age fetuses. Fetal Ther. 1988; 3: 158-164 [DOI:10.1159/000263348].

16. McIntire DD, Bloom SL, Casey BM, et al. Birth weight in relation to morbidity and mortality among newborn infants. N Engl J Med. 1999; 340: 1234-1238 [DOI:10.1056/NEJM199904223401603].

17. Sutherland AE, Crossley KJ, Allison BJ, et al. The effects of intrauterine growth restriction and antenatal glucocorticoids on ovine fetal lung development. Pediatr Res. 2012; 71: 689-696 [DOI:10.1038/pr.2012.19].

18. Miller SL, Chai M, Loose J, et al. The effects of maternal betamethasone administration on the intrauterine growth-restricted fetus. Endocrinology. 2007; 148: 1288-1295 [DOI:10.1210/en.2006-1058].

19. Simchen MJ, Alkazaleh F, Adamson SL, et al. The fetal cardiovascular response to antenatal steroids in severe early-onset intrauterine growth restriction. Am J Obstet Gynecol. 2004; 190: 296-304 [DOI:10.1016/j.ajog.2003.08.011].

20. Mori R, Kusuda S, Fujimura M, et al. Antenatal corticosteroids promote survival of extremely preterm infants born at 22 to 23 weeks of gestation. J Pediatr. 2011; 159: 110-114 [DOI:10.1016/j.jpeds.2010.12.039]. 